\title{
Exponential Dichotomies, the Fredholm Alternative, and Transverse Homoclinic Orbits in Partial Functional Differential Equations
}

\author{
Shigui Ruan ${ }^{1,3}$ and Weinian Zhang $^{2}$ \\ Received March 3, 2003; revised August 6, 2005
}

\begin{abstract}
In this paper we investigate the transversality of homoclinic orbits in partial functional differential equations. We first discuss the exponential dichotomies for linear operator equations. Then we show that the Fredholm Alternative holds if the homogeneous equation has exponential dichotomies on $\mathbf{R}$. Transversality of homoclinic orbits for periodically perturbed partial functional differential equations is studied using the Liapunov-Schmidt method and the Melnikov integral.
\end{abstract}

KEY WORDS: Exponential dichotomy; Liapunov-Schmidt method; Melnikov integral; Partial functional differential equations; The Fredholm Alternative; Transverse homoclinic orbit.

AMS SUBJECT CLASSIFICATIONS:35R10; 58F14.

\section{INTRODUCTION}

Exponential dichotomies have played an important role in studying various types (ordinary, parabolic, functional) of differential equations. For ordinary differential equations (see Coppel [8]), under certain assumptions, a linear system admits an exponential dichotomy in $[0, \infty)$ if and only if the inhomogeneous linear system has a bounded solution on $[0, \infty)$ for every bounded function. The theory of exponential dichotomies in finite

\footnotetext{
${ }^{1}$ Department of Mathematics, University of Miami, P. O. Box 249085, Coral Gables, FL 33124-4250, USA. E-mail: ruan@math.miami.edu

${ }^{2}$ Department of Mathematics, Sichuan University, Chengdu, Sichuan 610064, China. E-mail: matzwn@sohu.com

${ }^{3}$ To whom correspondence should be addressed.
} 
dimensional systems has been studied extensively, we refer to a series of papers by Sacker and Sell [22] and the monograph by Coppel [8].

If the linear homogeneous equation has exponential dichotomies in $(-\infty, 0]$ and $[0, \infty)$, then the Fredholm Alternative holds in the case of bounded solutions for the nonhomogeneous equation. This result in finite dimensional systems can be found in $[16,17,20]$, etc. The theory of exponential dichotomies and the Fredholm Alternative have also been generalized to infinite dimensional systems, for example, to functional differential equations by Lin [12], to parabolic equations by Blazquez [3], Zeng [27], and Zhang [28], and to abstract evolution equations by Chow and Leiva [7], Sacker and Sell [23], and Rodrigues and Ruas-Filho [19]. For further details on exponential dichotomies and properties of solutions to abstract equations, we refer to the monograph by Chicone and Latushkin [4].

Exponential dichotomies also arise in the linear variational equation at a homoclinic orbit of an autonomous equation. Generalizing the ideas in Chow et al. [6], Palmer [16] employed exponential dichotomies of the linear variational system to establish the existence of transversal homoclinic points in periodically perturbed systems. The procedure uses the Fredholm Alternative and Liapunov-Schmidt method associated with the Melnikov integral (see also Battelli and Lazzari [1], Battelli and Palmer [2], Meyer and Sell [15], and the references cited therein). Following the above mentioned techniques Blazquez [3], Zeng [27] and Zhang and Stewart [29] studied transverse homoclinc orbits in parabolic equations, Lin [12, 13] and Zhang and $\mathrm{Wu}[30]$ investigated transverse homoclinic orbits in functional differential equations.

Recently, great attention has been paid to the study of partial functional differential equations, we refer to the classical papers by Travis and Webb [24, 25], Martin and Smith [14], and the monograph by Wu [26]. Ruan et al. [21] studied the bifurcation from a homoclinic orbit in partial functional differential equations using Šilnikov's techniques. In this paper, we first discuss the exponential dichotomies for the linear operator equation. Then the Fredholm Alternative is established for the inhomogeneous operator equation. Finally, using Liapunov-Schmidt method and Melnikov integral, we study the transversality of homoclinic orbits in a periodically perturbed partial functional differential equation.

\section{EXPONENTIAL DICHOTOMIES}

Let $X$ denote a Banach space over $\mathbf{R}=(-\infty, \infty), J \subset \mathbf{R}$ an interval, and $B(J, X)$ the Banach space of bounded continuous $X$-valued functions from $J$ to $X$ equipped with the supremum norm. Let $r>0$ be a given constant and $\mathscr{C}=C([-r, 0] ; X)$ the Banach space of continuous $X$-valued 
functions on $[-r, 0]$ with the supremum norm $|\cdot|$. For any real numbers $a \leqslant b, t \in[a, b]$ and any continuous mapping $u:[a-r, b] \rightarrow X, u_{t}$ denotes the element of $\mathscr{C}$ given by $u_{t}(\theta)=u(t+\theta)$ for $\theta \in[-r, 0]$.

Consider the following linear operator equation

$$
\dot{u}(t)=A u(t)+L(t) u_{t},
$$

where $A$ is the infinitesimal generator of an analytic compact semigroup $\{S(t)\}_{t} \geqslant 0$ on $X$. For a function $\eta(t, \cdot):[-r, 0] \rightarrow B(X, X)$ of bounded variation for $t \in \mathbf{R}$, the linear operator $L(t): \mathscr{C} \rightarrow X$ is defined by

$$
L(t) \xi=\int_{-r}^{0}\left[\mathrm{~d}_{\theta} \eta(t, \theta)\right] \xi(\theta), \quad \xi \in \mathscr{C} .
$$

For $\phi \in \mathscr{C}$, denote the solution of Eq. (2.1) with initial value

$$
u_{s}=\phi
$$

by $u_{t}(s, \phi), t \geqslant s-r$. Define

$$
T(t, s) \phi=u_{t}(s, \phi) .
$$

Then $\{T(t, s)\}_{t \geqslant s}$ is an evolution family of bounded linear operators on $\mathscr{C}$ (see [24]).

Definition 2.1. It is said that $T(t, s)$ has an exponential dichotomy on an interval $J \subset \mathbf{R}$ with constants $K \geqslant 0$ (called the bound) and $\alpha>0$ (called the exponent) if there exist projections $P(\theta)$ and $Q(\theta)=I-P(\theta)$, strongly continuous in $\theta \in J$, such that

(i) $T(t, s) P(s)=P(t) T(t, s)$ for $t \geqslant s$ in $J$;

(ii) $\left.T(t, s)\right|_{\mathscr{R}(Q(s))}, t \geqslant s$, is an isomorphism from $\mathscr{R}(Q(s)$ ) onto $\mathscr{R}(Q(t))$ and $T(s, t): \mathscr{R}(Q(t)) \rightarrow \mathscr{R}(Q(s))$ is defined as the inverse of $\left.T(t, s)\right|_{\mathscr{R}(Q(s))}$, where $\mathscr{R}(Q(s))$ denotes the range of $Q(s)$;

(iii) $|T(t, s) P(s)| \leqslant K e^{-\alpha(t-s)}$ for $t \geqslant s$ in $J$;

(iv) $|T(t, s) Q(s)| \leqslant K e^{\alpha(t-s)}$ for $t \leqslant s$ in $J$.

$\mathscr{R}(P(t))$ and $\mathscr{R}(Q(t))$ are called the stable and unstable subspaces of $T(t, s)$, respectively. Since $T(t, s)$ is compact for $t>s+r$ (see Travis and Webb [25]), we assume that $\mathscr{R}(Q(t))$ is finite dimensional.

The adjoint of $\mathscr{C}$ is identified with $\mathscr{C}^{*}=C\left([0, r] ; X^{*}\right)$, where $X^{*}$ is the dual space of $X$ with the duality $\langle x, y\rangle$ for $x \in X$ and $y \in X^{*}$. For $\phi \in \mathscr{C}$ and $\psi \in \mathscr{C}^{*}$, we use the bilinear pairing

$$
\langle\langle\psi, \phi\rangle\rangle=\int_{-r}^{0}\langle\mathrm{~d} \psi(\theta), \phi(\theta)\rangle
$$


as defined in (3.3) on page 176 of Hale and Lunel [10], which differs from that one

$$
\langle\langle\psi, \phi\rangle\rangle=\langle\psi(0), \phi(0)\rangle-\int_{-r}^{0} \int_{0}^{\theta}\langle\psi(\xi-\theta)[\mathrm{d} \eta(\theta)], \phi(\xi)\rangle \mathrm{d} \xi
$$

in [24]. Let $T^{*}(s, t)$ be the adjoint of $T(t, s)$ defined by

$$
\langle\langle\psi, T(t, s) \phi\rangle\rangle=\left\langle\left\langle T^{*}(s, t) \psi, \phi\right\rangle\right\rangle, \quad \phi \in \mathscr{C}, \quad \psi \in \mathscr{C}^{*} .
$$

Then $T^{*}(s, t)$ is a linear semigroup for $s \leqslant t \in J$ and is weak* continuous with respect to $s \leqslant t \in J$. If $P^{*}(t)$ is the adjoint of $P(t)$, then it is a projection operator and is weak* continuous in $t \in J$. We can show that $T^{*}(s, t)$ has an exponential dichotomy on $J$ with weak* continuity.

Proposition 2.2. If $T(t, s)$ has an exponential dichotomy on $J \subset \mathbf{R}$ with constants $K \geqslant 0, \alpha>0$ and projections $P$ and $Q$, then the adjoint $T^{*}(s, t)$ satisfies

(i) $\quad T^{*}(s, t) P^{*}(t)=P^{*}(s) T^{*}(s, t)$ for $s \leqslant t$ in $J$;

(ii)' $\left.T^{*}(s, t)\right|_{\mathscr{R}\left(Q^{*}(t)\right)}, s \leqslant t$, is an isomorphism from $\mathscr{R}\left(Q^{*}(t)\right)$ onto $\mathscr{R}\left(Q^{*}(s)\right)$ and $T^{*}(t, s): \mathscr{R}\left(Q^{*}(s)\right) \rightarrow \mathscr{R}\left(Q^{*}(t)\right)$ is defined as the inverse of $\left.T^{*}(s, t)\right|_{\mathscr{R}\left(Q^{*}(t)\right)}$;

(iii) $\left|T^{*}(s, t) P^{*}(t)\right| \leqslant K e^{-\alpha(t-s)}$ for $s \leqslant t$ in $J$;

(iv) $\left|T^{*}(s, t) Q^{*}(t)\right| \leqslant K e^{\alpha(t-s)}$ for $s \geqslant t$ in $J$.

Proof. We only prove (ii)', the other statements are consequences of the definition of exponential dichotomy.

We first show that $\left.T^{*}(s, t)\right|_{\mathscr{R}\left(Q^{*}(t)\right)}: \mathscr{R}\left(Q^{*}(t)\right) \rightarrow \mathscr{R}\left(Q^{*}(s)\right)$ is one-toone. Choose $\xi \in \mathscr{R}\left(Q^{*}(t)\right)$ such that $T^{*}(s, t) \xi=0$. For any $\phi \in \mathscr{C}$, write $\phi=\phi_{1}+\phi_{2}$ with $\phi_{1} \in \mathscr{R}(Q(t))$ and $\phi_{2} \in \mathscr{R}(P(t))$. We have

$$
\langle\langle\xi, \phi\rangle\rangle=\left\langle\left\langle\xi, \phi_{1}+\phi_{2}\right\rangle\right\rangle=\left\langle\left\langle\xi, \phi_{1}\right\rangle\right\rangle+\left\langle\left\langle\xi, \phi_{2}\right\rangle\right\rangle=\left\langle\left\langle\xi, \phi_{1}\right\rangle\right\rangle .
$$

Now, there exists $\psi_{1} \in \mathscr{R}(Q(s))$ such that $\phi_{1}=T(t, s) \psi_{1}$. It follows that

$$
\left\langle\left\langle\xi, \phi_{1}\right\rangle\right\rangle=\left\langle\left\langle\xi, T(t, s) \psi_{1}\right\rangle\right\rangle=\left\langle\left\langle T^{*}(s, t) \xi, \psi_{1}\right\rangle\right\rangle=0,
$$

which implies that $\xi=0$ and $\left.T^{*}(s, t)\right|_{\mathscr{R}\left(Q^{*}(t)\right)}$ is one-to-one.

To prove that $\left.T^{*}(s, t)\right|_{\mathscr{R}\left(Q^{*}(t)\right)}$ is onto, fix $\eta \in \mathscr{R}\left(Q^{*}(s)\right)$. For any $\phi \in \mathscr{C}$, again write $\phi=\phi_{1}+\phi_{2}$ with $\phi_{1} \in \mathscr{R}(Q(t))$ and $\phi_{2} \in \mathscr{R}(P(t))$. Let $\psi_{1}$ be such that $T(t, s) \psi_{1}=\phi_{1}$. Define $\xi \in \mathscr{C}^{*}$ such that

$$
\langle\langle\xi, \phi\rangle\rangle=\left\langle\left\langle\eta, \psi_{1}\right\rangle\right\rangle, \quad \forall \phi \in \mathscr{C} .
$$

By the definition of exponential dichotomy it follows that $\xi$ is well defined. Also, since for $\phi \in \mathscr{C},\left\langle\left\langle P^{*}(t) \xi, \phi\right\rangle\right\rangle=\langle\langle\xi, P(t) \phi\rangle\rangle=0$, we have $\xi \in \mathscr{R}\left(Q^{*}(t)\right)$. 
Let $\psi=\psi_{1}+\psi_{2} \in \mathscr{C}$ with $\psi_{1} \in \mathscr{R}(Q(s))$ and $\psi_{2} \in \mathscr{R}(P(s))$. Then

$$
\left\langle\left\langle T^{*}(s, t) \xi, \psi\right\rangle\right\rangle=\langle\langle\xi, T(t, s) \psi\rangle\rangle=\left\langle\left\langle\xi, T(t, s) \psi_{1}+T(t, s) \psi_{2}\right\rangle\right\rangle
$$

with $T(t, s) \psi_{1} \in \mathscr{R}(Q(t))$ and $T(t, s) \psi_{2} \in \mathscr{R}(P(t))$. Let $\phi=T(t, s) \psi$. By the definition of $\xi$, it follows that

$$
\left\langle\left\langle T^{*}(s, t) \xi, \psi\right\rangle\right\rangle=\langle\langle\xi, \phi\rangle\rangle=\left\langle\left\langle\eta, \psi_{1}\right\rangle\right\rangle
$$

Notice that $\left\langle\left\langle\eta, \psi_{2}\right\rangle\right\rangle=\left\langle\left\langle\eta, P(s) \psi_{2}\right\rangle\right\rangle=\left\langle\left\langle P^{*}(s) \eta, \psi_{2}\right\rangle\right\rangle$. We thus have

$$
\left\langle\left\langle T^{*}(s, t) \xi, \psi\right\rangle\right\rangle=\left\langle\left\langle\eta, \psi_{1}\right\rangle\right\rangle=\left\langle\left\langle\eta, \psi_{1}\right\rangle\right\rangle+\left\langle\left\langle\eta, \psi_{2}\right\rangle\right\rangle=\langle\langle\eta, \psi\rangle\rangle
$$

for any $\psi \in \mathscr{C}$. Therefore, $T^{*}(s, t) \xi=\eta$. This completes the proof.

\section{THE FREDHOLM ALTERNATIVE}

In this section, we consider the following nonhomogeneous equation

$$
\dot{u}(t)=A u(t)+L(t) u_{t}+h(t)
$$

associated with the initial value $u_{s}=\phi$, where $h: \mathbf{R} \rightarrow X$ is continuous. Associate with the nonhomogeneous equation (3.1), we also consider the adjoint equation of Eq. (2.1) ([9])

$$
\dot{v}(s)=-A^{*} v(s)-L^{*}(s) v^{s},
$$

where $A^{*}$ is the adjoint of $A, v^{s} \in \mathscr{C}^{*}$ is given by $v^{s}(\tau)=v(s+\tau)$ for $\tau \in[0, r], L^{*}(s) \psi=\int_{-r}^{0}\left[\mathrm{~d}_{\alpha} \eta^{*}(s, \alpha)\right] \psi(-\alpha)$ for $\psi \in \mathscr{C}^{*}$ and $\eta^{*}$ is the adjoint of $\eta$. We note that $T^{*}\left(s, t_{0}\right)$ is the evolution operator of (3.2) and $v^{s}:=$ $T^{*}\left(s, t_{0}\right) v^{0}$ for each fixed $v^{0} \in \mathscr{C}^{*}$ satisfying (3.2). In fact, for each fixed $u_{0} \in \mathscr{C}$ Eq. (2.1) has the solution $u_{s}=T\left(s, t_{0}\right) u_{0}$ and

$$
\begin{aligned}
& \frac{\mathrm{d}}{\mathrm{d} s}\left\langle\left\langle v^{s}, u_{s}\right\rangle\right\rangle=\frac{\mathrm{d}}{\mathrm{d} s}\left\langle\left\langle T^{*}\left(s, t_{0}\right) v^{0}, u_{s}\right\rangle\right\rangle=\frac{\mathrm{d}}{\mathrm{d} s}\left\langle\left\langle v^{0}, T\left(t_{0}, s\right) u_{s}\right\rangle\right\rangle \\
& \quad=\frac{\mathrm{d}}{\mathrm{d} s}\left\langle\left\langle v^{0}, u_{0}\right\rangle\right\rangle=0, \quad \forall s .
\end{aligned}
$$

On the other hand, by (2.5) the left-hand side of (3.3) can be calculated as follows: 


$$
\begin{aligned}
\frac{\mathrm{d}}{\mathrm{d} s}\left\langle\left\langle v^{s}, u_{s}\right\rangle\right\rangle= & \frac{\mathrm{d}}{\mathrm{d} s} \int_{-r}^{0}\left\langle\mathrm{~d}_{\theta} v^{s}(\theta), u_{s}(\theta)\right\rangle \\
= & \int_{-r}^{0}\left\langle\mathrm{~d}_{\theta} \frac{\mathrm{d}}{\mathrm{d} s} v(\theta+s), u(\theta+s)\right\rangle+\int_{-r}^{0}\left\langle\mathrm{~d}_{\theta} v(\theta+s), \frac{\mathrm{d}}{\mathrm{d} s} u(\theta+s)\right\rangle \\
= & \int_{-r}^{0}\left\langle\mathrm{~d}_{\theta} \frac{\mathrm{d}}{\mathrm{d} s} v(\theta+s), u(\theta+s)\right\rangle \\
& +\int_{-r}^{0}\left\langle\mathrm{~d}_{\theta} v(\theta+s), A u(\theta+s)+L(\theta+s) u_{\theta+s}\right\rangle \\
= & \int_{-r}^{0}\left\langle\mathrm{~d}_{\theta} \frac{\mathrm{d}}{\mathrm{d} s} v(\theta+s), u(\theta+s)\right\rangle \\
& +\int_{-r}^{0}\left\langle\mathrm{~d}_{\theta}\left[A^{*} v(\theta+s)+L^{*}(\theta+s) v^{\theta+s}\right], u(\theta+s)\right\rangle
\end{aligned}
$$

because the just defined $L^{*}(s)$ satisfies

$$
\left\langle v(t), L(t) u_{t}\right\rangle=\left\langle L^{*}(t) v^{t}, u(t)\right\rangle, \quad \forall u(t) \in X, \quad \forall v(t) \in X^{*},
$$

as implied by Proposition 4.13 of Travis and Webb [24]. Hence $\frac{\mathrm{d}}{\mathrm{d} s} v(s)+$ $A^{*} v(s)+L^{*}(s) v^{s}=0$ by the arbitrary choice of $u_{0}$.

By Proposition 2.2, if the homogeneous equation (2.1) has exponential dichotomies on $\mathbf{R}_{+}=(0, \infty)$ and $\mathbf{R}_{-}=(-\infty, 0)$ with projections $P_{+}(t)$ and $P_{-}(t)$, respectively, then its adjoint equation (3.2) also has exponential dichotomies with the same exponents and bounds on $\mathbf{R}_{+}$and $\mathbf{R}_{-}$with projections $Q_{+}^{*}(t)$ and $Q_{-}^{*}(t)$, respectively, where $Q_{+}^{*}(t)=I-$ $P_{+}^{*}(t), Q_{-}^{*}(t)=I-P_{-}^{*}(t), P_{+}^{*}(t)$, and $P_{-}^{*}(t)$ are the adjoints of $P_{+}(t)$ and $P_{-}(t)$, respectively.

Definition 3.1. We say that the Fredholm Alternative holds if for each $h \in B(\mathbf{R}, X)$, the nonhomogeneous equation (3.1) has a bounded solution in $B(\mathbf{R}, X)$ if and only if

$$
\int_{-\infty}^{\infty}\langle v(t), h(t)\rangle \mathrm{d} t=0
$$

for every solution $v(t) \in B\left(\mathbf{R}, X^{*}\right)$ of the adjoint equation (3.2).

Define a map $X_{0}:[-r, 0] \rightarrow B(X, X)$ by

$$
X_{0}(\theta)= \begin{cases}0, & -r \leqslant \theta<0 \\ I, & \theta=0\end{cases}
$$


We have the following variation of constants formula for the nonhomogeneous equation (3.1) (Wu [26])

$$
u_{t}=T(t, s) \phi+\int_{s}^{t} T(t, \alpha) X_{0} h(\alpha) d \alpha .
$$

Following Theorem 7.6.3 in [11] and Theorem 4.2.2 in [26], we have the following proposition

Proposition 3.2. If the homogeneous equation (2.1) has an exponential dichotomy on $\mathbf{R}_{+}$with projections $P_{+}$and $Q_{+}$for any $h \in B\left(\mathbf{R}_{+}, X\right)$, then the nonhomogeneous Eq. (3.1) has a solution $u \in B\left(\mathbf{R}_{+}, X\right)$ if and only if

$$
\begin{aligned}
u_{t}= & T(t, 0) P_{+}(0) \phi+\int_{0}^{t} T(t, \alpha) P_{+}(\alpha) X_{0} h(\alpha) \mathrm{d} \alpha \\
& -\int_{t}^{\infty} T(t, \alpha) Q_{+}(\alpha) X_{0} h(\alpha) \mathrm{d} \alpha, \quad t \in \mathbf{R}_{+}
\end{aligned}
$$

for some $\phi \in \mathscr{C}$. Similarly, if (2.1) has an exponential dichotomy on $\mathbf{R}_{-}$with projections $P_{-}$and $Q_{-}$for any $h \in B\left(\mathbf{R}_{-}, X\right)$, then the nonhomogeneous $E q$. (3.1) has a solution $u \in B\left(\mathbf{R}_{-}, X\right)$ if and only if

$$
\begin{aligned}
u_{t}= & T(t, 0) Q_{-}(0) \phi+\int_{-\infty}^{t} T(t, \alpha) P_{-}(\alpha) X_{0} h(\alpha) \mathrm{d} \alpha \\
& +\int_{0}^{t} T(t, \alpha) Q_{-}(\alpha) X_{0} h(\alpha) \mathrm{d} \alpha, \quad t \in \mathbf{R}_{-}
\end{aligned}
$$

for some $\phi \in \mathscr{C}$.

We are now in a position to state and prove our main result in this section.

Theorem 3.3. (Fredholm alternative). Let $L(t)$ be continuous and bounded in the operator norm with respect to $t \in \mathbf{R}$. Suppose that the homogeneous equation (2.1) has exponential dichotomies on $\mathbf{R}_{+}$and $\mathbf{R}_{-}$with projections $P_{+}$and $P_{-}$, respectively. Define $F: B(\mathbf{R}, X) \rightarrow B(\mathbf{R}, X)$ by

$$
(F u)(t)=\frac{\mathrm{d}}{\mathrm{d} t} u(t)-A u(t)-L(t) u_{t} .
$$

Then $F$ is a Fredholm operator of index

$$
\operatorname{Ind} F=\operatorname{dim} \mathscr{R}\left(Q_{-}(0)\right)-\operatorname{dim} \mathscr{R}\left(Q_{+}(0)\right)
$$


and

$$
\begin{aligned}
\mathscr{R}(F)= & \{h \in B(\mathbf{R}, X): \\
& \left.\int_{-\infty}^{+\infty}\langle v(t), h(t)\rangle \mathrm{d} t=0 \text { for all solutions } v(t) \in B\left(\mathbf{R}, X^{*}\right) \text { of (3.2) }\right\}, \\
\mathscr{N}(F)= & \left\{[T(t, 0) \phi](0): \phi \in \mathscr{R}\left(P_{+}(0)\right) \cap \mathscr{R}\left(Q_{-}(0)\right), t \in \mathbf{R}\right\} .
\end{aligned}
$$

Proof. Since $\mathscr{N}(F)$ consists of all bounded solutions $u(t)=$ $[T(t, 0) \phi](0)$ of the homogeneous equation $(2.1)$, it follows by the exponential dichotomies of (2.1) on $\mathbf{R}_{ \pm}$that $T(t, 0) \phi \in B(\mathbf{R}, X)$ if and only if $\phi \in \mathscr{R}\left(P_{+}(0)\right) \cap \mathscr{R}\left(Q_{-}(0)\right)$. This shows that (3.11) holds.

For any $h(t) \in \mathscr{R}(F)$, there is a differentiable mapping $u(t) \in B(\mathbf{R}, X)$ such that

$$
h(t)=(F u)(t)=\frac{\mathrm{d}}{\mathrm{d} t} u(t)-A u(t)-L(t) u_{t} .
$$

Thus, each solution $v(t) \in B\left(\mathbf{R}, X^{*}\right)$ of the adjoint equation (3.2) satisfies

$$
\begin{aligned}
\langle v(t), h(t)\rangle & =\left\langle v(t), \frac{\mathrm{d}}{\mathrm{d} t} u(t)\right\rangle-\langle v(t), A u(t)\rangle-\left\langle v(t), L(t) u_{t}\right\rangle \\
& =\left\langle v(t), \frac{\mathrm{d}}{\mathrm{d} t} u(t)\right\rangle-\left\langle A^{*} v(t), u(t)\right\rangle-\left\langle L^{*}(t) v^{t}, u(t)\right\rangle \\
& =\left\langle v(t), \frac{\mathrm{d}}{\mathrm{d} t} u(t)\right\rangle+\left\langle\frac{d}{d t} v(t), u(t)\right\rangle \\
& =\frac{\mathrm{d}}{\mathrm{d} t}\langle v(t), u(t)\rangle .
\end{aligned}
$$

Since the adjoint equation (3.2) has an exponential dichotomy, it follows that $v(t) \rightarrow 0$ exponentially as $t \rightarrow \pm \infty$. By (3.13) and the boundedness of $h(t)$, we have

$$
\int_{-\infty}^{\infty}\langle v(t), h(t)\rangle \mathrm{d} t=\left.\langle v(t), u(t)\rangle\right|_{-\infty} ^{\infty}=0 .
$$

The sufficient conditions for $h \in \mathscr{R}(F)$ are that there exist $\phi \in \mathscr{C}$ and a continuous map $t \rightarrow u_{t}$ such that (3.7) and (3.8) hold, which have a solution $\phi \in \mathscr{C}$ if and only if

$$
\begin{aligned}
{\left[P_{+}(0)-Q_{-}(0)\right] \phi=} & \int_{-\infty}^{0} T(0, \alpha) P_{-}(\alpha) X_{0} h(\alpha) \mathrm{d} \alpha \\
& +\int_{0}^{\infty} T(0, \alpha) Q_{+}(\alpha) X_{0} h(\alpha) \mathrm{d} \alpha
\end{aligned}
$$


Since $P_{+}(0)$ is a Fredholm operator and $Q_{-}(0)$ is compact, it follows that $P_{+}(0)-Q_{-}(0)$ is a Fredholm operator. Thus, $\mathscr{R}\left(P_{+}(0)-Q_{-}(0)\right)$ is closed and (3.15) has a solution $\phi \in \mathscr{C}$ if and only if

$$
\left\langle\left\langle\psi,\left[P_{+}(0)-Q_{-}(0)\right] \phi\right\rangle\right\rangle=0
$$

for all $\psi \in \mathscr{N}\left(\left(P_{+}(0)-Q_{-}(0)\right)^{*}\right)=\mathscr{N}\left(P_{+}^{*}(0)-Q_{-}^{*}(0)\right)$. However, for $\psi \in$ $\mathscr{N}\left(P_{+}^{*}(0)-Q_{-}^{*}(0)\right)$, we have

$$
\psi_{0} \equiv P_{-}^{*}(0) \psi=Q_{+}^{*}(0) \psi \in \mathscr{R}\left(P_{-}^{*}(0)\right) \cap \mathscr{R}\left(Q_{+}^{*}(0)\right)
$$

Since the adjoint equation (3.2) has an exponential dichotomy, it follows that $\left[T^{*}(t, 0) \psi_{0}\right](0)$ is a bounded solution of (3.2) and approaches 0 exponentially as $t \rightarrow \pm \infty$. Also

$$
\begin{aligned}
& \left\langle\left\langle\psi,\left[P_{+}(0)-Q_{-}(0)\right] \phi\right\rangle\right\rangle \\
= & \int_{-\infty}^{0}\left\langle\left\langle\psi, T(0, \alpha) P_{-}(\alpha) X_{0} h(\alpha)\right\rangle\right\rangle \mathrm{d} \alpha+\int_{0}^{\infty}\left\langle\left\langle\psi, T(0, \alpha) Q_{+}(\alpha) X_{0} h(\alpha)\right\rangle\right\rangle \mathrm{d} \alpha \\
= & \int_{-\infty}^{0}\left\langle\left\langle T^{*}(\alpha, 0) P_{-}^{*}(0) \psi, X_{0} h(\alpha)\right\rangle\right\rangle \mathrm{d} \alpha+\int_{0}^{\infty}\left\langle\left\langle T^{*}(\alpha, 0) Q_{+}^{*}(0) \psi, X_{0} h(\alpha)\right\rangle\right\rangle \mathrm{d} \alpha \\
= & \int_{-\infty}^{0}\left\langle\left\langle T^{*}(\alpha, 0) \psi_{0}, X_{0} h(\alpha)\right\rangle\right\rangle \mathrm{d} \alpha+\int_{0}^{\infty}\left\langle\left\langle T^{*}(\alpha, 0) \psi_{0}, X_{0} h(\alpha)\right\rangle\right\rangle \mathrm{d} \alpha \\
= & \int_{-\infty}^{\infty}\left\langle\left\langle T^{*}(\alpha, 0) \psi_{0}, X_{0} h(\alpha)\right\rangle\right\rangle \mathrm{d} \alpha \\
= & 0
\end{aligned}
$$

if $\int_{-\infty}^{\infty}\langle v(t), h(t)\rangle \mathrm{d} t=0$ for all bounded solution $v(t)$ of the adjoint equation (3.2). Thus, the characterization (3.11) of $\mathscr{R}(F)$ is proved.

To prove that $\mathscr{R}(F)$ is closed, $\operatorname{dim} \mathscr{N}(F)<\infty$, and codim $\mathscr{R}(F)<\infty$, define $J: \mathscr{R}\left(P_{-}^{*}(0)\right) \cap \mathscr{R}\left(Q_{+}^{*}(0)\right) \rightarrow B^{*}(\mathbf{R}, X)$ as follows:

$$
J\left(\psi_{0}\right)(h)=\int_{-\infty}^{\infty}\left\langle\left\langle T^{*}(\alpha, 0) \psi_{0}, X_{0} h(\alpha)\right\rangle\right\rangle \mathrm{d} \alpha
$$

for any $\psi \in \mathscr{R}\left(P_{-}^{*}(0)\right) \cap \mathscr{R}\left(Q_{+}^{*}(0)\right)$ and $h \in B(\mathbf{R}, X)$. We can see that $J$ is linear and one-to-one. Thus, $\mathscr{R}\left(P_{-}^{*}(0)\right) \cap \mathscr{R}\left(Q_{+}^{*}(0)\right) \cong J\left(\mathscr{R}\left(P_{-}^{*}(0)\right) \cap\right.$ $\left.\mathscr{R}\left(Q_{+}^{*}(0)\right)\right) \subset B^{*}(\mathbf{R}, X)$. The characterization (3.11) of $\mathscr{R}(F)$ implies that $\mathscr{R}(F) \subset B(\mathbf{R}, X)$ is an annihilator of $\left(\mathscr{R}\left(P_{-}^{*}(0)\right) \cap \mathscr{R}\left(Q_{+}^{*}(0)\right)\right)$, that is,

$$
\mathscr{R}(F)=\bigcap\left\{\mathscr{N}(\bar{\psi}): \bar{\psi} \in J\left(\mathscr{R}\left(P_{-}^{*}(0)\right) \cap \mathscr{R}\left(Q_{+}^{*}(0)\right)\right)\right\} .
$$


It follows that $\mathscr{R}(F)$ is closed and

$$
\begin{aligned}
\operatorname{dim} \mathscr{N}(F) & =\operatorname{dim} \mathscr{R}\left(P_{-}(0)\right) \cap \mathscr{R}\left(Q_{+}(0)\right) \\
& \leqslant \operatorname{dim}^{\prime} \mathscr{R}\left(Q_{+}(0)\right) \\
& <\infty \\
\operatorname{codim} \mathscr{R}(F) & =\operatorname{dim} J\left(\mathscr{R}\left(P_{-}^{*}(0)\right) \cap \mathscr{R}\left(Q_{+}^{*}(0)\right)\right) \\
& =\operatorname{dim} \mathscr{R}\left(P_{-}^{*}(0)\right) \cap \mathscr{R}\left(Q_{+}^{*}(0)\right) \\
& \leqslant \operatorname{dim} \mathscr{R}\left(Q_{+}^{*}(0)\right) \\
& <\infty .
\end{aligned}
$$

Therefore, $F$ is a Fredholm operator. Finally, following Zhang [28] we can establish the index formula (3.10) for the operator $F$. This completes the proof.

\section{TRANSVERSE HOMOCLINIC ORBITS}

In this section, we consider the following nonlinear equation

$$
\dot{u}(t)=A u(t)+g\left(u_{t}\right) .
$$

Assume that

(H1) $g \in C^{k}(\mathscr{C}, X), k \geqslant 1$, and $g(0)=0$.

(H2) Equation (4.1) has a hyperbolic equilibrium $u_{0} \in X$ and a homoclinic orbit $p(t) \in X$ connecting $u_{0}$ (i.e., $\left\|p(t)-u_{0}\right\| \rightarrow 0$ as $t \rightarrow \pm \infty$ ).

Since $u_{0}$ is a hyperbolic equilibrium, the linearized equation at $u_{0}$

$$
\dot{u}(t)=A u(t)+g^{\prime}\left(u_{0}\right) u_{t}
$$

admits an exponential dichotomy on R. Moreover, since

$$
\left\|p(t)-u_{0}\right\| \rightarrow 0 \quad \text { as } t \rightarrow \pm \infty
$$

the roughness of exponential dichotomies (see Pliss and Sell [18]) implies that the linearized equation along the homoclinic orbit $p(t)$,

$$
\dot{u}(t)=A u(t)+g^{\prime}\left(p_{t}\right) u_{t},
$$

has exponential dichotomies on $\mathbf{R}_{+}$and $\mathbf{R}_{-}$with projections $P_{+}(t)$ and $P_{-}(t)$, respectively, and $\operatorname{dim} Q_{+}(0)=\operatorname{dim} Q_{-}(0)$. We should note that the variational equation (4.2) does not have exponential dichotomies on $\mathbf{R}$ since (4.2) has at least a bounded solution $\dot{p}(t)$ on $\mathbf{R}$. We thus assume that 
(H3) $\dot{p}(t)$ is the unique bounded solution (counting constant multiples) of the linearized equation (4.2).

By Theorem 3.3, the operator

$$
(G u)(t)=\frac{\mathrm{d} u}{\mathrm{~d} t}-A u-g^{\prime}\left(p_{t}\right) u_{t}
$$

is a Fredholm operator with $\operatorname{Ind}(G)=0$. On the other hand, assumption (H3) implies that the adjoint equation of (4.2) has a unique bounded solution $v(t)$. Assume that

$$
\int_{-\infty}^{\infty} v^{2}(t) \mathrm{d} t=1
$$

Now consider the perturbed equation

$$
\dot{u}(t)=A u(t)+g\left(u_{t}\right)+\varepsilon h\left(t, u_{t}, \varepsilon\right) .
$$

Assume that

(H4) $h \in C^{k}(\mathbf{R} \times \mathscr{C} \times \mathbf{R}, X), k \geqslant 1$, is $T$-periodic in $t, h(t, 0,0)=0, \varepsilon \in \mathbf{R}$ is a parameter.

(H5) The Melnikov function

$$
M(\beta)=\int_{-\infty}^{\infty}\left\langle v(t), h\left(t+\beta, p_{t}, 0\right)\right\rangle d t
$$

has a simple zero $\beta_{0}$, i.e., $M\left(\beta_{0}\right)=0$ but $M^{\prime}\left(\beta_{0}\right) \neq 0$.

The main result in this section is the following theorem.

Theorem 4.1. If the assumptions (HI)-(H5) hold, then

(i) for $\varepsilon$ sufficiently small, Eq. (4.4) has a unique hyperbolic T-periodic solution $u_{0}(t, \varepsilon) \in X$ satisfying $u_{0}(t, 0)=u_{0}$;

(ii) for $\varepsilon \neq 0$ sufficiently small there exists a bounded solution $u^{*}(t, \varepsilon) \in X$ such that

$$
\left\|u^{*}(t, \varepsilon)-u_{0}(t, \varepsilon)\right\| \rightarrow 0 \quad \text { as } \varepsilon \rightarrow 0
$$

(iii) the linearized equation at $u^{*}(t, \varepsilon)$,

$$
\dot{w}(t)=A w(t)+\left[g^{\prime}\left(u_{t}^{*}\right)+\varepsilon \frac{\partial h}{\partial u}\left(t, u_{t}^{*}, \varepsilon\right)\right] w_{t},
$$

has an exponential dichotomy on $\mathbf{R}$. 
Proof. (i) We can see that the linearized equation at $u_{0}$,

$$
\dot{u}(t)=A u(t)+g^{\prime}\left(u_{0}\right) u_{t},
$$

has an exponential dichotomy on $\mathbf{R}$. Regarding $u_{0}(t) \equiv u_{0}$ as a T-periodic solution, since it is a trivial periodic solution, it follows that 1 is not in the spectrum of the period map for the linearized Eq. (4.4) for $u_{0}(t), \varepsilon=0$. Hence, Theorem 8.3.1 of Henry [11] implies that for $\varepsilon$ sufficiently small, the perturbed Eq. (4.4) has a unique T-periodic solution $u_{0}(t, \varepsilon) \in X$ satisfying $u_{0}(t, 0)=u_{0}$.

(ii) Make a change of variable in a neighborhood of $p(t)$ by

$$
z(t)=u(t+\beta)-p(t), \quad \beta \in \mathbf{R} .
$$

Then Eq. (4.4) becomes

$$
\dot{z}(t)=A z(t)+g^{\prime}\left(p_{t}\right) z_{t}+H\left(t, z_{t}, \beta, \varepsilon\right),
$$

where

$$
\begin{aligned}
H\left(t, z_{t}, \beta, \varepsilon\right)= & \varepsilon h\left(t+\beta, z_{t}(\beta, \varepsilon)+p_{t}, \varepsilon\right) \\
& +g\left(z_{t}(\beta, \varepsilon)+p_{t}\right)-g\left(p_{t}\right)-g^{\prime}\left(p_{t}\right) z_{t}(\beta, \varepsilon) .
\end{aligned}
$$

Since the linear part of (4.6) (i.e. Eq. (4.2)) admits exponential dichotomies on both $\mathbf{R}_{+}$and $\mathbf{R}_{-}$, an application of the Liapunov-Schmidt method (Chow and Hale [5]) implies that Eq. (4.6) is equivalent to the following two equations

$$
\begin{aligned}
\dot{z}(t)= & A z(t)+g^{\prime}\left(p_{t}\right) z_{t}+H\left(t, z_{t}, \beta, \varepsilon\right) \\
& -v(t) \int_{-\infty}^{\infty}\left\langle v(t), H\left(t, z_{t}, \beta, \varepsilon\right)\right\rangle \mathrm{d} t, \\
& \int_{-\infty}^{\infty}\left\langle v(t), H\left(t, z_{t}, \beta, \varepsilon\right)\right\rangle d t=0 .
\end{aligned}
$$

Following Battelli and Palmer [2] or Zhang [28] (using the Implicit Function Theorem), for $\beta-\beta_{0}$ and $\varepsilon$ sufficiently small Eq. (4.7) has a unique bounded solution $z=z(t, \beta, \varepsilon)$ such that $z\left(t, \beta_{0}, 0\right)=0$. Moreover, $z(t, \beta, \varepsilon)$ is smooth in $t, \beta$, and $\varepsilon$. Substituting $z(t, \beta, \varepsilon)$ into (4.8), we obtain the following bifurcation equation

$$
\begin{aligned}
G(\beta, \varepsilon)= & \int_{-\infty}^{\infty}\left\langle v(t), H\left(t, z_{t}, \beta, \varepsilon\right)\right\rangle \mathrm{d} t \\
= & \int_{-\infty}^{\infty}\left\langle v(t), \varepsilon h\left(t+\beta, z_{t}(\beta, \varepsilon)+p_{t}, \varepsilon\right)\right. \\
& \left.+g\left(z_{t}(\beta, \varepsilon)+p_{t}\right)-g\left(p_{t}\right)-g^{\prime}\left(p_{t}\right) z_{t}(\beta, \varepsilon)\right\rangle \mathrm{d} t .
\end{aligned}
$$


We have

$$
\begin{aligned}
G_{\varepsilon}(\beta, \varepsilon)= & \int_{-\infty}^{\infty}\left\langle v(t), h\left(t+\beta, z_{t}(\beta, \varepsilon)+p_{t}, \varepsilon\right)+\varepsilon \frac{\mathrm{d}}{\mathrm{d} \varepsilon} h\left(t+\beta, z_{t}(\beta, \varepsilon)+p_{t}, \varepsilon\right)\right. \\
& \left.+g^{\prime}\left(z_{t}(\beta, \varepsilon)+p_{t}\right) \frac{\mathrm{d}}{\mathrm{d} \varepsilon}\left[z_{t}(\beta, \varepsilon)+p_{t}\right]-g^{\prime}\left(p_{t}\right) \frac{\mathrm{d}}{\mathrm{d} \varepsilon}\left[z_{t}(\beta, \varepsilon)+p_{t}\right]\right\rangle \mathrm{d} t
\end{aligned}
$$

and

$$
\begin{aligned}
G_{\beta}(\beta, \varepsilon)= & \int_{-\infty}^{\infty}\left\langle v(t), \varepsilon \frac{\mathrm{d}}{\mathrm{d} \beta} h\left(t+\beta, z_{t}(\beta, \varepsilon)+p_{t}, \varepsilon\right)\right. \\
& \left.+g^{\prime}\left(z_{t}(\beta, \varepsilon)+p_{t}\right) \frac{\mathrm{d}}{\mathrm{d} \beta}\left[z_{t}(\beta, \varepsilon)+p_{t}\right]-g^{\prime}\left(p_{t}\right) \frac{\mathrm{d}}{\mathrm{d} \beta}\left[z_{t}(\beta, \varepsilon)+p_{t}\right]\right\rangle \mathrm{d} t .
\end{aligned}
$$

Define

$$
\tilde{G}(\beta, \varepsilon)=\left\{\begin{array}{l}
G(\beta, \varepsilon) / \varepsilon, \quad \varepsilon \neq 0, \\
G_{\varepsilon}(\beta, 0), \quad \varepsilon=0 .
\end{array}\right.
$$

By assumption (H5), we know that

$$
\tilde{G}\left(\beta_{0}, 0\right)=M\left(\beta_{0}\right)=0, \quad \tilde{G}_{\beta}\left(\beta_{0}, 0\right)=M^{\prime}\left(\beta_{0}\right) \neq 0 .
$$

Thus, the Implicit Function Theorem implies that for $\varepsilon$ sufficiently small, there exists a continuously differentiable function $\beta=\beta(\varepsilon)$ with $\beta(0)=\beta_{0}$, such that $\tilde{G}(\beta(\varepsilon), \varepsilon)=0$. Hence, $G(\beta(\varepsilon), \varepsilon)=0$, i.e., the bifurcation equation (and thus (4.3)) has a unique bounded solution $u^{*}(t, \varepsilon)$ with

$$
u^{*}(t+\beta(\varepsilon), \varepsilon)=z(t, \beta(\varepsilon), \varepsilon)+p(t) .
$$

Obviously,

$$
\lim _{\varepsilon \rightarrow 0} u^{*}(t, \varepsilon)=p(t)
$$

Since

$$
\left\|u^{*}(t, \varepsilon)-u_{0}(t, \varepsilon)\right\| \leqslant\left\|u^{*}(t, \varepsilon)-p(t)\right\|+\left\|p(t)-u_{0}(t, \varepsilon)\right\|,
$$

we have

$$
\left\|u^{*}(t, \varepsilon)-u_{0}(t, \varepsilon)\right\| \rightarrow 0 \quad \text { as } \varepsilon \rightarrow 0 .
$$

(iii) Notice that to show that for sufficiently small $\varepsilon \neq 0$ Eq. (4.5) has an exponential dichotomy on $\mathbf{R}$ is equivalent to show that for sufficiently small $\varepsilon \neq 0$ the equation 


$$
\dot{w}(t)=A w(t)+\left[g^{\prime}\left(z_{t}+p_{t}\right)+\varepsilon \frac{\partial}{\partial u} h\left(t+\beta(\varepsilon), z_{t}+p_{t}, \varepsilon\right)\right] w_{t}
$$

has an exponential dichotomy on $\mathbf{R}$. For fixed $t$ and $\varepsilon$, define $A(t, \varepsilon): \mathscr{C} \rightarrow X$ by

$$
A(t, \varepsilon) \phi=(A \phi)(0)+\left[g^{\prime}\left(z_{t}+p_{t}\right)+\varepsilon \frac{\partial}{\partial u} h\left(t+\beta(\varepsilon), z_{t}+p_{t}, \varepsilon\right)\right] \phi, \quad \phi \in \mathscr{C} .
$$

We can see that $A(t, 0) \phi=(A \phi)(0)+g^{\prime}\left(p_{t}\right) \phi$. Since (4.2) has exponential dichotomies on $\mathbf{R}_{+}$and $\mathbf{R}_{-}$with projections $P_{+}(t)$ and $P_{-}(t)$, respectively, and $\operatorname{dim} Q_{+}(0)=\operatorname{dim} Q_{-}(0)$. The roughness of exponential dichotomies ([23]) implies that for sufficiently small $\varepsilon \neq 0$ Eq. (4.12) also has an exponential dichotomy on $\mathbf{R}_{+}$and $\mathbf{R}_{-}$with projections $P_{+}^{\varepsilon}(t)$ and $P_{-}^{\varepsilon}(t)$, respectively. In order to show that Eq. (4.12) has an exponential dichotomy on $\mathbf{R}$, it suffices to define

$$
\begin{aligned}
X_{1}(t) & = \begin{cases}\mathscr{R}\left(P_{+}^{\varepsilon}(t)\right), & t \geqslant 0, \\
\left\{\phi: T_{\varepsilon}(0, t) \phi \in \mathscr{R}\left(P_{+}^{\varepsilon}(0)\right)\right\}, & t \leqslant 0,\end{cases} \\
X_{2}(t) & = \begin{cases}\mathscr{R}\left(Q_{-}^{\varepsilon}(t)\right), & t \leqslant 0, \\
\left\{T_{\varepsilon}(t, 0) \phi: \phi \in \mathscr{R}\left(Q_{-}^{\varepsilon}(0)\right)\right\}, & t \geqslant 0,\end{cases}
\end{aligned}
$$

where $T_{\varepsilon}(t, s)$ denotes the evolution operator for (4.12), and claim that

$$
X_{1}(t) \oplus X_{2}(t)=X, \quad \forall t \in \mathbf{R} .
$$

First, we prove that (4.13) holds for $t=0$. For an arbitrary real number $\gamma$, the change of variable $y(t)=w(t)-\gamma \dot{p}(t)$ transforms the system

$$
\dot{w}(t)=A(t, \varepsilon) w_{t}+\varepsilon^{2} f(t)
$$

into

$$
\dot{y}(t)=A(t, 0) w_{t}+[A(t, \varepsilon)-A(t, 0)]\left(y_{t}+\gamma \dot{p}_{t}\right)+\varepsilon^{2} f(t),
$$

which is equivalent by the Liapunov-Schmidt reduction to the system

$$
\begin{gathered}
\int_{-\infty}^{\infty}\left\langle v(t),[A(t, \varepsilon)-A(t, 0)]\left(y_{t}+\gamma \dot{p}_{t}\right)+\varepsilon^{2} f(t)\right\rangle \mathrm{d} t=0, \\
\dot{y}(t)=A(t, 0) w_{t}+[A(t, \varepsilon)-A(t, 0)]\left(y_{t}+\gamma \dot{p}_{t}\right)+\varepsilon^{2} f(t)+v(t) \\
\times \int_{-\infty}^{\infty}\left\langle v(t),[A(t, \varepsilon)-A(t, 0)]\left(y_{t}+\gamma \dot{p}_{t}\right)+\varepsilon^{2} f(t)\right\rangle \mathrm{d} t,
\end{gathered}
$$

where $v(t)$ is given in $\mathscr{R}(F)$ in Theorem 3.3. One can prove that for small $\varepsilon$ Eq. (4.17) has a unique bounded solution $y=y(t ; \gamma, \varepsilon)$ such that 
$y(t ; \gamma, 0)=0$. Substituting $y=y(t ; \gamma, \varepsilon)$ in (4.16) we obtain the bifurcation equation

$$
B(\gamma, \varepsilon):=\int_{-\infty}^{\infty}\left\langle v(t),[A(t, \varepsilon)-A(t, 0)]\left(y_{t}(\gamma, \varepsilon)+\gamma \dot{p}_{t}\right)+\varepsilon^{2} f(t)\right\rangle \mathrm{d} t=0 .
$$

It is easy to check that $B(\gamma, \varepsilon)$ is $C^{2}$ and $B(\gamma, 0)=0$. Furthermore, define $H(\gamma, \varepsilon):=B(\gamma, \varepsilon) / \varepsilon$ for $\varepsilon \neq 0$ and $H(\gamma, \varepsilon):=\frac{\partial}{\partial \varepsilon} B(\gamma, 0)$ for $\varepsilon=0$. In order to solve the implicit function from $H(\gamma, \varepsilon)=0$, we observe that

$$
H(\gamma, 0)=\frac{\partial}{\partial \varepsilon} B(\gamma, 0)=\gamma \int_{-\infty}^{\infty}\left\langle v(t), \frac{\partial}{\partial \varepsilon} A(t, 0) \dot{p}_{t}\right\rangle \mathrm{d} t .
$$

Obviously $H(0,0)=0$. Moreover, $\frac{\partial}{\partial \varepsilon} A(t, 0)=g^{\prime \prime}\left(p_{t}\right) \frac{\partial}{\partial \varepsilon} z_{t}(t, 0)+\frac{\partial}{\partial u} h(t+$ $\left.\beta_{0}, p_{t}, 0\right)$. Notice that

$$
\dot{z}(t, \varepsilon)=A z(t, \varepsilon)+g\left(z_{t}+p_{t}\right)-g\left(p_{t}\right)+\varepsilon h\left(t+\beta(\varepsilon), z_{t}+p_{t}, \varepsilon\right) .
$$

Differentiating both sides of Eq. (4.20) with respect to $\varepsilon$ and setting $\varepsilon=0$, we have

$$
\frac{\partial}{\partial \varepsilon} \dot{z}(t, 0)=A \frac{\partial}{\partial \varepsilon} z(t, 0)+g^{\prime}\left(p_{t}\right) \frac{\partial}{\partial \varepsilon} z_{t}(t, 0)+h\left(t+\beta_{0}, p_{t}, 0\right) .
$$

Differentiating both sides of Eq. (4.21) with respect to $t$ yields

$$
\begin{aligned}
\frac{\mathrm{d}}{\mathrm{d} t} \frac{\partial}{\partial \varepsilon} \dot{z}(t, 0)= & A \frac{\partial}{\partial \varepsilon} \dot{z}(t, 0)+g^{\prime}\left(p_{t}\right) \frac{\partial}{\partial \varepsilon} \dot{z}_{t}(t, 0)+\frac{\partial}{\partial t} h\left(t+\beta_{0}, p_{t}, 0\right) \\
& +\left[g^{\prime \prime}\left(p_{t}\right) \frac{\partial}{\partial \varepsilon} z_{t}(t, 0)+\frac{\partial}{\partial u} h\left(t+\beta_{0}, p_{t}, 0\right)\right] \dot{p}_{t} \\
= & A \frac{\partial}{\partial \varepsilon} \dot{z}(t, 0)+g^{\prime}\left(p_{t}\right) \frac{\partial}{\partial \varepsilon} \dot{z}_{t}(t, 0) \\
& +\left[\frac{\partial}{\partial t} h\left(t+\beta_{0}, p_{t}, 0\right)+\frac{\partial}{\partial \varepsilon} A(t, 0) \dot{p}_{t}\right] .
\end{aligned}
$$

It follows that $\frac{\partial}{\partial \varepsilon} \dot{z}(t, 0) \in X$ is a bounded solution on $\mathbf{R}$ of the equation

$$
\dot{z}(t)=A z(t)+g^{\prime}\left(p_{t}\right) z_{t}+\left[\frac{\partial}{\partial t} h\left(t+\beta_{0}, p_{t}, 0\right)+\frac{\partial}{\partial \varepsilon} A(t, 0) \dot{p}_{t}\right] .
$$

By Theorem 3.3, we have

$$
\int_{-\infty}^{\infty}\left\langle v(t), \frac{\partial}{\partial t} h\left(t+\beta_{0}, p_{t}, 0\right)+\frac{\partial}{\partial \varepsilon} A(t, 0) \dot{p}_{t}\right\rangle \mathrm{d} t=0 .
$$


It follows from (4.19) that

$$
\begin{aligned}
\frac{\partial}{\partial \gamma} H(0,0) & =\int_{-\infty}^{\infty}\left\langle v(t), \frac{\partial}{\partial \varepsilon} A(t, 0) \dot{p}_{t}\right\rangle \mathrm{d} t \\
& =-\int_{-\infty}^{\infty}\left\langle v(t), \frac{\partial}{\partial t} h\left(t+\beta_{0}, p_{t}, 0\right)\right\rangle \mathrm{d} t \\
& =-M^{\prime}\left(\beta_{0}\right) \neq 0 .
\end{aligned}
$$

By the Implicit Function Theorem, there exists a continuous function $\gamma=$ $\gamma(\varepsilon)$ such that $\gamma(0)=0$ and $H(\gamma(\varepsilon), \varepsilon)=0$ for sufficiently small $\varepsilon$. From the bifurcation equation (4.18), we know that Eq. (4.14) has a bounded solution $w(t, \varepsilon)=y(t, \gamma(\varepsilon), \varepsilon)+\gamma(\varepsilon) \dot{p}(t)$. This implies that the operator $F_{\varepsilon}: w(t) \mapsto$ $\dot{w}(t)-A(t, \varepsilon) w_{t}$ has the range $\mathscr{R}\left(F_{\varepsilon}\right)=B(\mathbf{R}, X)$, i.e., $\operatorname{codim} \mathscr{R}\left(F_{\varepsilon}\right)=0$. By Theorem 3.3, index $\left(F_{\varepsilon}\right)=0$ for small $\varepsilon$. Thus $\operatorname{dim} \mathscr{N}\left(F_{\varepsilon}\right)=\operatorname{index}\left(F_{\varepsilon}\right)+$ $\operatorname{codim} \mathscr{R}\left(F_{\varepsilon}\right)=0$ and therefore, $\mathscr{R}\left(P_{+}^{\varepsilon}(0)\right) \cap \mathscr{R}\left(Q_{-}^{\varepsilon}(0)\right)=\mathscr{N}\left(F_{\varepsilon}\right)=\{0\}$. It follows from Lemma 4 in [28] that

$$
\begin{aligned}
& \operatorname{codim}\left(\mathscr{R}\left(P_{+}^{\varepsilon}(0)\right)+\mathscr{R}\left(Q_{-}^{\varepsilon}(0)\right)\right) \\
& \quad=\operatorname{codim} \mathscr{R}\left(P_{+}^{\varepsilon}(0)\right)-\operatorname{dim} \mathscr{R}\left(Q_{-}^{\varepsilon}(0)\right)+\operatorname{dim}\left(\mathscr{R}\left(P_{+}^{\varepsilon}(0)\right) \cap \mathscr{R}\left(Q_{-}^{\varepsilon}(0)\right)\right) \\
& \quad=\operatorname{codim} \mathscr{R}\left(P_{+}^{\varepsilon}(0)\right)-\operatorname{dim} \mathscr{R}\left(Q_{+}^{\varepsilon}(0)\right)=0,
\end{aligned}
$$

implying $\mathscr{R}\left(P_{+}^{\varepsilon}(0)\right)+\mathscr{R}\left(Q_{-}^{\varepsilon}(0)\right)=X$, i.e., (4.13) holds for $t=0$.

Next, we show that

$$
X_{1}(t)+X_{2}(t)=X, \quad \forall t \in \mathbf{R}
$$

For $t \geqslant 0$ we have $X_{1}(t)=\mathscr{R}\left(P_{+}^{\varepsilon}(t)\right), X_{2}(t)=\left\{T_{\varepsilon}(t, 0) \phi: \phi \in \mathscr{R}\left(Q_{-}^{\varepsilon}(0)\right)\right\}$ and $\operatorname{dim} X_{2}(t)=\operatorname{dim} X_{2}(0)$ since $T_{\varepsilon}(t, 0)$ is an isomorphism of $\mathscr{R}\left(Q_{-}^{\varepsilon}(0)\right)$ onto $\mathscr{R}\left(Q_{-}^{\varepsilon}(t)\right)$. From (4.13), for $t=0$ we also know that $\operatorname{codim} X_{1}(t)=$ $\operatorname{codim} X_{1}(0)$. Therefore (4.23) holds for $t \geqslant 0$. For $t \leqslant 0$ and $\phi \in X$, we know $T(0, t) \phi=\phi_{1}+\phi_{2}$ by (4.13) for $t=0$, where $\phi_{1} \in X_{1}(0), \phi_{2} \in X_{2}(0)$. By the definition of $X_{1}(t)$ and $X_{2}(t)$ there exists $\tilde{\phi}_{1} \in X_{1}(t)$ such that $\phi_{1}=$ $T_{\varepsilon}(0, t) \tilde{\phi}_{1}$. Thus $\phi_{2}=T_{\varepsilon}(0, t)\left(\phi-\tilde{\phi}_{1}\right)$. The fact that $\phi_{2} \in X_{2}(0)$ implies that $\phi-\tilde{\phi}_{1} \in \mathscr{R}\left(Q_{-}^{\varepsilon}(t)\right)$. Hence, there exists $\tilde{\phi}_{2} \in X_{2}(t)$ such that $\phi=\tilde{\phi}_{1}+\tilde{\phi}_{2}$, implying (4.23) for $t \leqslant 0$. Furthermore, we can also prove that $X_{1}(t) \cap$ $X_{2}(t)=\{0\}$ for all $t \in \mathbf{R}$. This proves (4.13) for all $t \in \mathbf{R}$.

Thus, for sufficiently small $\varepsilon \neq 0$, the linearized equation (4.5) has an exponential dichotomy on $\mathbf{R}$. This completes the proof of Theorem 4.1.

Remark 4.2. The existence of an exponential dichotomy of Eq. (4.5) on $\mathbf{R}$ implies that the bounded solution $u^{*}(t, \varepsilon)$ is a transversal orbit homoclinic to the hyperbolic $T$-periodic solution $u_{0}(t, \varepsilon)$. 
An example can be given by modifying the example in [12], which is the partial functional differential equation

$$
\begin{aligned}
\frac{\partial}{\partial t} u(x, t)= & -(\operatorname{ch} 1)(\operatorname{sh} 1)^{-1} \frac{\partial^{2}}{\partial x^{2}} u(x, t)-(\operatorname{sh} 1)^{-1}\left(1+\left(\frac{u(x, t)}{\sin x}\right)^{2}\right) u(x, t-1) \\
& +\varepsilon \cos t
\end{aligned}
$$

Associated with the boundary value condition

$$
u(0, t)=u(\pi, 0)=0,
$$

Eq. (4.24) explicitly has a homoclinic orbit $u(x, t)=(\operatorname{sh} 1) \sin x(\operatorname{ch} t)^{-1}$ when $\varepsilon=0$. Note that $(\operatorname{sh} 1) \sin x(\operatorname{ch} t)^{-1} \rightarrow 0$ as $t \rightarrow \pm \infty$ and the spectrum of the operator $A:=-(\operatorname{ch} 1)(\operatorname{sh} 1)^{-1} \frac{\partial^{2}}{\partial x^{2}}$ consists of the simple eigenvalues $\lambda_{j}=-(\operatorname{ch} 1)(\operatorname{sh} 1)^{-1} j^{2}, j=1,2, \ldots$ Hence, system (4.24) for $\varepsilon=0$ has a hyperbolic equilibrium $u_{0}=0 \in X:=H_{0}^{1}(0, \pi) \cap H^{2}(0, \pi)$. In order to apply Theorem 4.1 we need to compute the Melnikov function

$$
M(\beta)=\int_{-\infty}^{\infty}\left\langle v_{0}(t), \cos (t+\beta)\right\rangle \mathrm{d} t
$$

in condition (H5), where $v_{0}(t)$ is an nontrivial solution of the adjoint system. Although numerical calculation of $v_{0}(t)$ was illustrated in [12], there are still the usual computational difficulties associated with $M(\beta)$ since the spaces $X$ and $\mathscr{C}$ are infinitely dimensional.

\section{ACKNOWLEDGMENTS}

The authors would like to thank the referee and Professor George Sell for their helpful comments and suggestions on the early versions of this paper. Research was partially supported by NSF grant DMS-0412047 and the University of Miami. (S.R.) by NSFC(China) grant 10471101, TRAPOYT and the China MOE Research Grants (W.Z.).

\section{REFERENCES}

1. Battelli, F., and Lazzari, C. (1990). Exponential dichotomies, heteroclinic orbits, and Melnikov functions, J. Differential Equations 86, 342-366.

2. Battelli, F., and Palmer, K. J. (1993). Chaos in the Duffing equation, J. Differential Equations 101, 276-301.

3. Blazquez, C. M. (1986). Transverse homoclinic orbits in periodically perturbed parabolic equations, Nonlinear Analysis - TMA 10, 1277-1291.

4. Chicone, C., and Latushkin, Y. (1999). Evolution Semigroups in Dynamical Systems and Differential Equations, Mathematical Survey Monograph, Vol. 70, American Mathematic Society, Providence. 
5. Chow, S.-N., and Hale, J. K. (1982). Methods of Bifurcations, Springer-Verlag, Berlin.

6. Chow, S.-N., Hale, J. K., and Mallet-Paret, J. (1980). An example of bifurcation to homoclinic orbits, J. Differential Equations 37, 351-373.

7. Chow, S.-N., and Leiva, H. (1996). Unbounded perturbation of the exponential dichotomy for evolution equations, J. Differential Equations 129, 509-531.

8. Coppel, W. A. (1978). Dichotomies in Stability Theory, Lecture Notes in Mathematical, Vol. 629, Springer-Verlag, New York.

9. Faria, T., Huang, W., and Wu, J., (2002). Smoothness of center manifolds for maps and formal adjoints for semilinear FDEs in general Banach spaces, SIAM J. Math. Anal. 34, 173-203.

10. Hale, J. K., and Verduyn Lunel, S. M. (1993). Introduction to Functional Differential Equations, Springer-Verlag, New York.

11. Henry, D. (1981). Geometric Theory of Semilinear Parabolic Equations, Lecture Notes in Mathematical, Vol. 840, Springer-Verlag, New York.

12. Lin, X. B. (1986). Exponential dichotomies and homoclinic orbits in functional differential equations, J. Differential Equations 63, 227-254.

13. Lin, X. B. (1990). Using Melnikov's method to solve Šilnikov's problems, Proc. Roy. Soc. Edinburgh Sect. A 116, 295-325.

14. Martin, R., and Smith, H. L. (1990). Abstract functional differential equations and reaction-diffusion system, Trans. Amer. Math. Soc. 321, 1-44.

15. Meyer, K. R., and Sell, G. R. (1989). Melnikov transforms, Bernoulli bundles and almost periodic perturbations, Trans. Amer. Math. Soc. 314, 63-105.

16. Palmer, K. J. (1984). Exponential dichotomies and transversal homoclinic points, J. Differential Equations 55, 225-256.

17. Palmer, K. J. (1988). Exponential dichotomies and Fredholm operators, Proc. Amer. Math. Soc. 104, 149-156.

18. Pliss, V. A., and Sell, G. R. (1999). Robustness of exponential dichotomies in infinitedimensional dynamical systems, J. Dynam. Differential Equations 11, 471-513.

19. Rodrigues, H. M., and Ruas-Filho, J. G. (1995). Evolution equations: dichotomies and the Fredholm alternative for bounded solutions, J. Differential Equations 119, 263-283.

20. Rodrigues, H. M., and Silveira, M. (1988). On the relationship between exponential dichotomies and the Fredholm alternative, J. Differential Equations 79, 78-91.

21. Ruan, S., Wei, J., and Wu, J. (2003). Bifurcation from a homoclinic orbit in partial functional differential equations, Discrete Contin. Dyn. Syst. 9, 1293-1322.

22. Sacker, R. J., and Sell, G. R. (1974, 1976). Existence of dichotomies and invariant splittings for linear differential systems, I, II, III. J. Differential Equations 15, 429-458; 22, 478-496; 497-522.

23. Sacker, R. J., and Sell, G. R. (1994). Dichotomies for linear evolutionary equations in Banach spaces, J. Differential Equations 113, 17-67.

24. Travis, C. C., and Webb, G. F. (1974). Existence and stability for partial functional differential equations, Trans. Amer. Math. Soc. 200, 395-418.

25. Travis, C. C., and Webb, G. F. (1978). Existence, stability and compactness in the $\alpha$-norm for partial functional differential equations, Trans. Amer. Math. Soc. 240, 129-143.

26. Wu, J. (1996). Theory and Applications of Partial Functional Differential Equations, Springer-Verlag, New York.

27. Zeng, W. (1997). Transversality of homoclinic orbits and exponential dichotomies for parabolic equations, J. Math. Anal. Appl. 216, 466-480.

28. Zhang, W. (1995). The Fredholm alternative and exponential dichotomies for parabolic equations, J. Math. Anal. Appl. 191, 180-201. 
29. Zhang, W., and Stewart, I. (1996). Bounded solutions for non-autonomous parabolic equations, Dyn. Stability Syst. 11, 109-120.

30. Zhang, W., and Wu, J.-Y. (2000). Homoclinic orbits on invariant manifolds of a functional differential equation, J. Differential Equations 165, 414-429. 